\title{
Broadband Signal Spectrum Monitoring Method Based on Chirp-Z Transform
}

\author{
Yalan Song ${ }^{1, a}$, Zhibin Zeng ${ }^{2, b}$ \\ ${ }^{1}$ Communication University of China, Beijing, China \\ ${ }^{2}$ Communication University of China, Beijing, China \\ asongyalan0516@163.com, b'zhbzeng@cuc.edu.cn
}

\begin{abstract}
Keywords: Chirp-Z transform, broadband signal, sampling rate, spectrum analysis
Abstract. Sampling rate reduction is a key to spectrum analysis of broadband signals. To avoid spectrum aliasing, the sampling rate for broadband signal is always too high for implementation. In this paper, a new spectrum analyzing method based on respectively signal is proposed. Compared with conventional FFT, the Chirp-Z transform has the advantages of low sampling rate and high -resolution. Simulation results show that the Chirp-Z transform can achieve better spectrum analysis performance with the significant reduction on sampling rate.
\end{abstract}

\section{Introduction}

With the development of new media and mobile network, wireless communication systems are developing rapidly for transmission efficiency. Since the increase of transmission capacity, the bandwidth of wireless communication signals is becoming more and more wide. Moreover, when the broadband signal is amplified by the RF power amplifier, the output signal of the power amplifier will generally produce a spectrum expansion due to the nonlinearity of power amplifier ${ }^{[1]}$. And expansion of spectrum will demands higher sampling frequency and hardware costs. In LTE - A $100 \mathrm{MHz}$ signal, for example, the bandwidth output signal from power amplifier is extended to $500 \mathrm{MHz}$, so the sampling frequency of ADC sampling frequency is at least $1 \mathrm{GHz}$. To achieve such a high sampling frequency, the difficulty of the system design and the complexity of the hardware and algorithm are all challenges.

Spectrum analysis plays an important role in signal processing ${ }^{[2]}$. Different from the traditional signal spectrum analysis method of FFT, the Chirp-Z transform (CZT) focuses on signal analysis within a limited bandwidth, which will get higher resolution and more accuracy. Compared with FFT, Chirp z transform method has an important advantage to allow the spectrum aliasing, which significantly reduces the requirement for sampling rate.

\section{Chirp-Z Algorithm}

\subsection{The Basic Principle of CZT}

CZT sampling is carried out along a spiral trajectory instead of the unit circle within $\mathrm{Z}$ plane. In this case, the interested spectrum by dense samplingcan be calculated. Moreover, Chirp- $Z$ algorithm frequency resolution can be adjusted ${ }^{[3]}$, for the number of input points and the number of output can be unequal and the angle of the vectors of the sampling points can be arbitrary.

Assume that in a set of points $z_{k}$ calculate its value of $Z$ transformation, can be expressed as:

$$
X\left(z_{k}\right)=\sum_{n=0}^{N-1} x(n) z_{k}^{-n}, \quad k=0,1,2, \ldots L-1
$$

In Equation 1, x (n) is finite-length sequence of length $\mathrm{N}$. Analysis of $M$ points on the $Z$ plane spectrum sampling values, $z_{k}=A W^{k}(k=0 \sim M-1)$, therefore, the result by CZT can be expressed as Equation 2:

$$
X\left(z_{k}\right)=\sum_{n=0}^{N-1} x(n) A^{-n} W^{n k}(k=0 \sim M-1)
$$


In Equation 2, $A=A_{0} e^{j \theta_{0}}$ and $A_{0}$ is the radius of the initial sample vector $Z_{0}$, the angle $\theta_{0}=2 \pi f_{o} / f_{s} . W=W_{0} e^{-j \varphi_{0}}$ and $W_{0}$ is the trend of the path, $\varphi_{0}=2 \pi\left(f_{h}-f_{o}\right) /\left(M f_{s}\right)$, $f_{o}, f_{h}$ and $f_{s}$ demonstrate starting frequency, cut-off frequency and sampling rate, respectively. The rest of the sampling points can be obtained by sampling with $\varphi_{0}$ at the equal interval along the spiral line.

\subsection{Fast Algorithm of CZT}

According to the traditional method of CZT with the disadvantages of more complexity and low calculating efficiency, the formula $n k=\frac{1}{2}\left[n^{2}+k^{2}-(k-n)^{2}\right]$ can be used for equation 2 :

$$
X\left(z_{k}\right)=W^{k^{2} / 2} \sum_{n=0}^{N-1}\left[x(n) A^{-n} W^{n^{2} / 2}\right] W^{-(k-n)^{2} / 2}
$$

Substitute $g(n)=x(n) A^{-n} W^{n^{2} / 2}$ and $h(k-n)=W^{-(k-n)^{2} / 2}$ into the equation 3 and then the equation 4 can be obtained as follows:

$$
X\left(z_{k}\right)=W^{k^{2} / 2} \sum_{n=0}^{N-1} g(n) h(k-n)=W^{k^{2} / 2}[g(n) * h(n)]
$$

This transform is Bluestein algorithm ${ }^{[4]}$, and the convolution form of the CZT can be implemented by the above transformation and fast Fourier transform algorithm ${ }^{[5]}$. The block diagram of the CZT fast algorithm is shown in Fig.1.

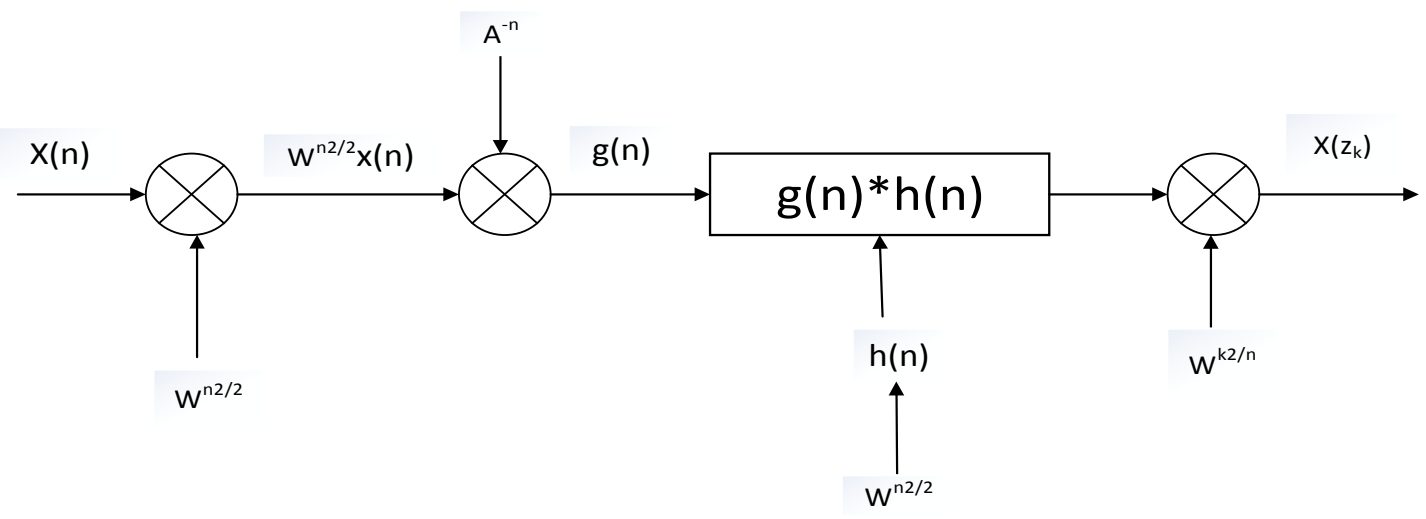

Figure.1 The block diagram of the CZT fast algorithm

\section{Application of Chirp Z measuring broadband signals}

\subsection{The technical index of the broadband signal spectrum monitoring}

There are two important technical index of the broadband signal spectrum monitoring: in-band spectrum ripple and in-band to sideband power ratio (ISPR).

In-band spectrum ripple is defined as the broadband within each frequency point relative to the center frequency amplitude variation. It depicts when the input signal amplitude is fixed, the law of the output signal's amplitude changes with frequency. There are different requirements in different systems. ISPR is a measure of the power amplifier linearity indicator, which is the ratio of the power value of the signal center frequency point and the power that deviates from the center frequency of the carrier outside of a point. As the extent of the power amplifier linearization is better, the value of ISPR is higher than the more. Generally ISPR of digital TV transmitter is more than 36dB.

Chirp z transform method can monitor the broadband signal in-band spectrum ripple and ISPR, the observed signal is within the desired range of technical indicators, if out of range, warning . 


\subsection{Simulation results of broadband signal spectrum measurement with Chirp-z method}

The input data used in this paper is from the XG100L-10D PA, and the sampling data points to 2048 points, working in nonlinear area, the input signal using OFDM bandwidth of $80 \mathrm{MHz}$, divided into I, $\mathrm{Q}$ two signals. Memory polynomial model of the power amplifier with memory depth $\mathrm{L}=2$ and memory order $\mathrm{K}=5$. The spectrum of input signal passing through the PA is shown in Fig. 2.

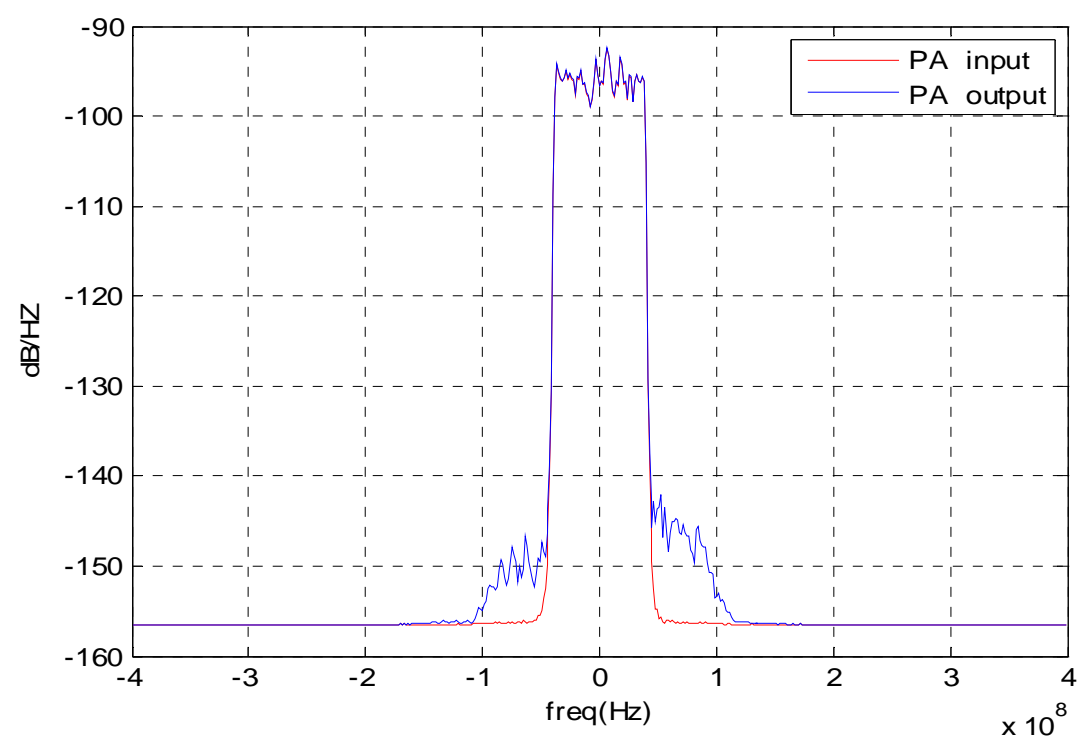

Figure. 2 Signal through the spectrum of the PA

$\mathrm{CZT}$ and FFT are performed for comparison, respectively with frequency range from for $13 \mathrm{MHz}$ to $14 \mathrm{MHz}$, as shown in Fig. 3.
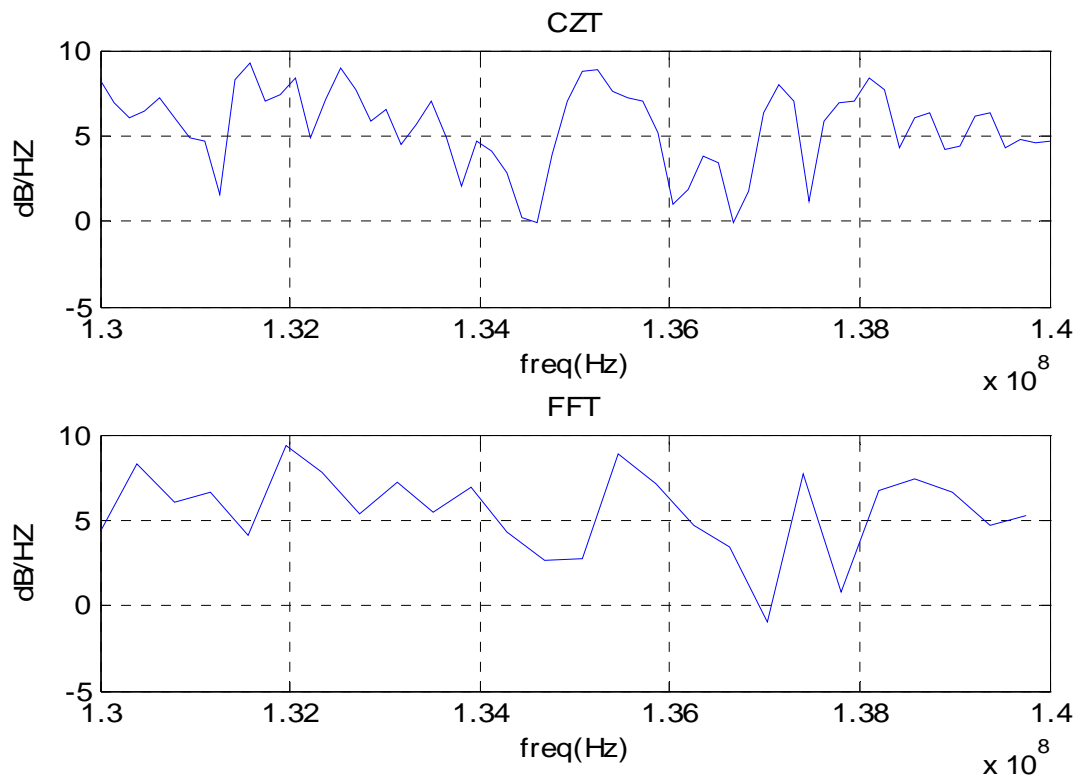

Figure.3 Spectrum diagram of PA sideband

The signal is divided into two paths (I and Q), and the signal spectrum of each path passing the amplifier will be extended to five times the bandwidth. For FFT algorithm the sampling rate should be as high as $400 \mathrm{MHz}$ to avoid aliasing, and however, the sampling rate of CZT is only $10 \mathrm{MHz}$. And it can be seen from Fig.3 that CZT can not only reduce the sampling rate but also able to reduce sampling rate for high frequency resolution. Since the "barrier effect" of FFT related to the length of the resolution and analysis, higher the accuracy ,longer the length of the sampling sequence. And this will result in more calculation and poor real-time performance. As a result, it can only be used for rough frequency spectrum analysis. For broadband signal, FFT also increase the sampling rate of signal, making it hard on hardware implementation. CZT can not only analyze our interested 
bandwidth spectrum with higher precision and resolution, but also the frequency spectrum with significant reduction on sampling rate. In general, using CZT is a method with high efficiency for broadband signal spectrum monitoring and measurement.

\section{Conclusions}

Spectrum analysis of broadband signal based on CZT can be summarized as follows:

1. Different from the traditional signal spectrum analysis method of FFT, Chirp-Z transform can analyze the spectrum of a narrow band range under the condition of without prolonging sampling length, and the resolution is high enough to achieve the effect of spectrum refinement.

2. The nonlinearity of the broadband power amplifier will lead to the spectrum expansion and increase the requirements for ADC. The Chirp- $\mathrm{Z}$ transform can be used to carry out spectrum monitoring with spectrum aliasing,and then reduces the hardware requirements.

3. Chirp-Z transform method is used in monitoring broadband signal spectrum, can select the part of the signal from the exciter directly in Chirp-z transform, which reduces the complexity of the spectrum measurement.

\section{References}

[1] D. Lei. H. H. Leung, X. P. Huang. Experimental Evaluation of Predistortion Tech. for High-Power Amplifer [J]. IEEE Trans. on Instr. and Meas., 2006, Vol. 55, No.6: 2155-2162.

[2] Xiangong Huang, Zhaohua Wang. Phase Difference Correcting Spectrum Method Based on All-phase Spectrum Analysis [J].Journal of Electronics and Information Technology, 2008,30(2):293-297. (In Chinese)

[3] L.R Rabiner, R.W Schafer, C.M. Rarer. The Chirp z-transform Algorithm. IEEE Transactions on Audio and Electroacoustics, 1969, 17(2):86-92.

[4] L .I. Bluestein. A liner filtering approach to the compotation of the discrete Fourier transform[C]. Northeast Electronics Research and Engineering Meeting Record, 1968, 10:218-219

[5] Kemao Qian, Chuanqi Li. New spectrum correction method based on Chirp Z Transform [J]. Journal of Vibration Engineering, 2000, 13(4):628-631. (In Chinese)

[6] Gough P T. A fast spectral estimation algorithm based on the FFT [J]. IEEE Transactions on Signal Processing, 1994, 42(6):1317-1322. 